\title{
NONVANISHING OF JACOBI POINCARÉ SERIES
}

\author{
SOUMYA DAS \\ (Received 4 October 2009; accepted 8 November 2010) \\ Communicated by F. Calegari
}

\begin{abstract}
We prove that, under suitable conditions, a Jacobi Poincaré series of exponential type of integer weight and matrix index does not vanish identically. For the classical Jacobi forms, we construct a basis consisting of the 'first' few Poincare series, and also give conditions, both dependent on and independent of the weight, that ensure the nonvanishing of a classical Jacobi Poincare series. We also obtain a result on the nonvanishing of a Jacobi Poincare series when an odd prime divides the index.
\end{abstract}

2010 Mathematics subject classification: primary 11F50; secondary 11F30.

Keywords and phrases: Jacobi forms, Poincaré series, Kloosterman sums.

\section{Introduction}

Rankin [9] proved that the $m$ th Poincare series $P_{m}^{k}$ of weight $k$ for the full modular group $\operatorname{SL}(2, \mathbb{Z})$ is not identically zero for sufficiently large positive integers $k$ and finitely many positive integers $m$ depending on $k$. Mozzochi extended Rankin's result to integral weight modular forms for congruence subgroups in [7].

In this paper we prove similar results for higher-degree Jacobi Poincaré series defined on the full Jacobi group $\Gamma_{g}^{J}=\operatorname{SL}(2, \mathbb{Z}) \ltimes\left(\mathbb{Z}^{g} \times \mathbb{Z}^{g}\right)$, where $g$ is a positive integer called the degree of the Jacobi group. The Jacobi group operates on $\mathcal{H} \times \mathbb{C}^{g}$ and also on functions $\phi: \mathcal{H} \times \mathbb{C}^{g} \rightarrow \mathbb{C}$, where $\mathcal{H}$ denotes the upper half plane. We write $\left.\right|_{k, m}$ for the action on functions. See Section 2 for the definitions.

Let $\operatorname{PSym}\left(g, \frac{1}{2} \mathbb{Z}\right)$ be the set of symmetric, positive definite, half integral $(g \times g)$ matrices. We define $A[B]:=B^{t} A B$ for matrices $A$ and $B$ of appropriate sizes, where $B^{t}$ is the transpose of the matrix $B$, and $e(z):=e^{2 \pi i z}$. More generally, we will use the standard notation $e_{a}(z):=e^{2 \pi i z / a}$.

Let $k, g \in \mathbb{Z}$, and $m \in \operatorname{PSym}\left(g, \frac{1}{2} \mathbb{Z}\right)$. The vector space of Jacobi cusp forms of weight $k$, index $m$ and degree $g$, denoted by $J_{k, m, g}^{\text {cusp }}$, is defined to be the space of all

(C) 2011 Australian Mathematical Publishing Association Inc. 1446-7887/2011 \$16.00 
holomorphic functions $\phi: \mathcal{H} \times \mathbb{C}^{g} \rightarrow \mathbb{C}$ that satisfy $\left.\phi\right|_{k, m} \gamma=\phi$, for all $\gamma \in \Gamma_{g}^{J}$, and have a Fourier expansion of the form

$$
\phi(\tau, z)=\sum_{\substack{n \in \mathbb{Z}^{+}, r \in \mathbb{Z}^{g} \\ 4 n>m^{-1}\left[r^{t}\right]}} c_{\phi}(n, r) e(n \tau+r z) .
$$

We denote $J_{k, m, 1}^{\text {cusp }}$ by $J_{k, m}^{\text {cusp }}$.

For $n \in \mathbb{Z}^{+}, r \in \mathbb{Z}^{g}$ and $m \in \operatorname{PSym}\left(g, \frac{1}{2} \mathbb{Z}\right)$ such that $4 n>m^{-1}\left[r^{t}\right]$, let $P_{n, r}^{k, m}$ be the $(n, r)$ th Poincaré series of weight $k$ and index $m$ (of exponential type) defined when $k>g+2$, as in [1] (see Section 2 for the definition). It is well known that the Poincaré series $P_{n, r}^{k, m}$, where $n \in \mathbb{Z}$ and $r \in \mathbb{Z}^{g}$, span $J_{k, m, g}^{\text {cusp }}$. It is then natural to ask whether such Poincaré series vanish identically or not. We prove the following theorem, which gives a partial answer to this question.

Let $k^{\prime}:=k-g / 2-1$ and define

$$
D:=\operatorname{det}\left(\begin{array}{cc}
2 n & r \\
r^{t} & 2 m
\end{array}\right) .
$$

THEOREM 1.1. Suppose that $2 r \equiv 0 \bmod \mathbb{Z}^{g} \cdot 2 m$. Then there exist an integer $k_{0}$ that depends only on $g$, and a constant $B>3 \log 2$ such that for all even $k \geq k_{0}$, the Jacobi Poincaré series $P_{n, r}^{k, m}$ does not vanish identically when

$$
k^{\prime} \leq \frac{\pi D}{\operatorname{det}(2 m)} \leq k^{\prime 1+\alpha(g)} \exp \left(-\frac{B \log k^{\prime}}{\log \log k^{\prime}}\right)
$$

where

$$
\alpha(g)= \begin{cases}\frac{2}{3(g+2)} & \text { if } 1 \leq g \leq 4, \\ \frac{2}{3 g} & \text { if } g \geq 5 .\end{cases}
$$

We construct a basis of $J_{k, m}^{\text {cusp }}$ consisting of the 'first' $\operatorname{dim}\left(J_{k, m}^{\text {cusp }}\right)$ Poincaré series (see Theorem 6.1 in Section 6). We also give conditions for the nonvanishing of Poincaré series independent of the weight for the classical Jacobi forms, where $g=1$.

As in [9], define

$$
M(x):=\exp \left(\frac{B_{1} \log x}{\log \log 2 x}\right) \quad \forall x \geq 2,
$$

where $B_{1}$ is a constant as in [9] $\left(B_{1}>\log 2\right)$.

THEOREM 1.2. Suppose that $g=1$ and $\pi D>2 m$. Then $P_{D, r}^{k, m} \not \equiv 0$ provided that

$$
M\left(\frac{\pi D}{m}\right) \sigma_{0}(D) D<\frac{m^{8 / 7}}{2^{9 / 4} \pi}\left(\frac{2}{6^{2 / 3}}+\frac{54}{2^{5 / 6}}+\frac{16}{2^{3 / 4}}\right)^{-3 / 2}
$$

where $\sigma_{0}(D):=\sum_{d \mid D} 1$. 
Finally, following [9], we give conditional statements on the nonvanishing of Jacobi Poincaré series, based on the relation between $g$-dimensional Kloosterman sums and the corresponding one-dimensional sums and identities involving these.

THEOREM 1.3. Suppose that $\mu \in \mathbb{Z}^{+}$and that $p$ is an odd prime such that $p \mid(m, r)$ but $p \nmid n$. If $P_{p^{\mu} n, p^{\mu}}^{k, p^{\mu} m} \not \equiv 0$, then either $P_{n p^{\mu-1}, r p^{\mu-1}}^{k, m p^{\mu-1}} \not \equiv$ or both $P_{n p^{2 \mu}, r p^{2 \mu}}^{k, p^{2 \mu}} \not \equiv 0$ and $P_{n, r p^{\mu}}^{k, p^{2 \mu} m} \not \equiv 0$.

(Here $p \mid m$ means that $p$ divides every entry of $m$; this makes sense since $2 m$ is a $(g \times g)$ matrix with integer entries and $p$ is odd.)

REMARK 1.4. In Section 3, we first prove that the Poincare series $P_{n, r}^{k, m}$ does not vanish when

$$
\frac{\pi D}{\operatorname{det}(2 m)}=2 \pi\left(n-\frac{1}{4} m^{-1}\left[r^{t}\right]\right) \leq C k^{\prime},
$$

giving the constant $C$ explicitly, and pointing out for which $k$ this is valid. This follows from Proposition 3.1 for arbitrary $g$ and also from Theorem 6.1 in the case where $g=1$ (recall that $\operatorname{dim} J_{k, m, 1}^{\text {cusp }} \leq C\left(\frac{1}{12} k(m+1)\right)$, where the constant $C$ may be taken to be 1 when $k$ is large enough).

REMARK 1.5. Theorem 1.1 improves the trivial case mentioned in the previous remark. However, achieving the 'order of $k^{2-\epsilon}$ when $\epsilon>0$ ' as in [9] in the case of Jacobi Poincaré series using Rankin's methods seems difficult, mainly because of the presence of the factor $\operatorname{gcd}(c, D)$ instead of $\operatorname{gcd}(c, D)^{1 / 2}$ in the estimate of Kloosterman sums of degree $g$ (even for small $g$ ); see Section 3.

REMARK 1.6. The condition that $k$ be even when $2 r \equiv 0 \bmod \mathbb{Z}^{g} \cdot 2 m$ in Theorem 1.1 is necessary, as the $(n, r)$ th Poincaré series vanish when $k$ is odd and $2 r \equiv 0$ $\bmod \mathbb{Z}^{g} \cdot 2 m$. The restriction $k^{\prime} \leq \pi D / \operatorname{det}(2 m)$ in Theorem 1.1 is natural, since we know the result in the complement (see Proposition 3.1). The same is true for the condition $\pi D>2 m$ in Theorem 1.2.

\section{Notation and preliminaries}

The Jacobi group $\Gamma_{g}^{J}$ operates on $\mathcal{H} \times \mathbb{C}^{g}$ in the usual way by

$$
\left(\left(\begin{array}{ll}
a & b \\
c & d
\end{array}\right),(\lambda, \mu)\right) \circ(\tau, z):=\left(\frac{a \tau+b}{c \tau+d},(c \tau+d)^{-1}(z+\lambda \tau+\mu)\right) .
$$

Let $k \in \mathbb{Z}$ and $m \in \operatorname{PSym}\left(g, \frac{1}{2} \mathbb{Z}\right)$. Then the action of $\Gamma_{g}^{J}$ on functions $\phi: \mathcal{H} \times \mathbb{C}^{g} \rightarrow \mathbb{C}$ is given by

$$
\begin{gathered}
\left.\phi\right|_{k, m} \gamma(\tau, z):=(c \tau+d)^{-k} e\left(-c(c \tau+d)^{-1} m[z+\lambda \tau+\mu]\right. \\
\left.+m[\lambda] \tau+2 \lambda^{t} m z\right) \phi(\gamma \circ(\tau, z)) .
\end{gathered}
$$


The vector space of Jacobi cusp forms of weight $k$, index $m$ and degree $g$, denoted by $J_{k, m, g}^{\text {cusp }}$, is defined to be the space of all holomorphic functions $\phi: \mathcal{H} \times \mathbb{C}^{g} \rightarrow \mathbb{C}$ that satisfy $\left.\phi\right|_{k, m} \gamma=\phi$, for all $\gamma \in \Gamma_{g}^{J}$, and have a Fourier expansion of the form

$$
\phi(\tau, z)=\sum_{\substack{n \in \mathbb{Z}^{+}, r \in \mathbb{Z}^{g} \\ 4 n>m^{-1}\left[r^{t}\right]}} c_{\phi}(n, r) e(n \tau+r z) .
$$

For $n \in \mathbb{Z}^{+}$and $r \in \mathbb{Z}^{g}$ such that $4 n>m^{-1}\left[r^{t}\right]$, let $P_{n, r}^{k, m}$ be the $(n, r)$ th Poincaré series of weight $k$ and index $m$ (of exponential type) defined when $k>g+2$ by

$$
P_{n, r}^{k, m}(\tau, z):=\left.\sum_{\gamma \in \Gamma_{g, \infty}^{J} \backslash \Gamma_{g}^{J}} e(n \tau+r z)\right|_{k, m} \gamma(\tau, z) \quad \forall \tau \in \mathcal{H}, z \in \mathbb{C}^{g},
$$

where

$$
\Gamma_{g, \infty}^{J}:=\left\{\left(\left(\begin{array}{ll}
1 & n \\
0 & 1
\end{array}\right),(0, \mu)\right) \mid n \in \mathbb{Z}, \mu \in \mathbb{Z}^{g}\right\} .
$$

It is well known that $J_{k, m, g}^{\text {cusp }}$ is finite-dimensional and the family of Poincare series $P_{n, r}^{k, m}$, where $n \in \mathbb{Z}^{+}$and $r \in \mathbb{Z}^{g}$, generate the space $J_{k, m, g}^{\text {cusp }}$. In [1, Lemma 1], Böcherer and Kohnen found the following Fourier expansion of $P_{n, r}^{k, m}$.

Proposition 2.1. The function $P_{n, r}^{k, m}$ is in $J_{k, m, g}^{\mathrm{cusp}}$. The Fourier expansion of the Poincaré series is given by

$$
P_{n, r}^{k, m}(\tau, z)=\sum_{\substack{n^{\prime} \in \mathbb{Z}^{+}, r^{\prime} \in \mathbb{Z}^{g} \\ 4 n^{\prime}>m^{-1}\left[r^{\prime \prime}\right]}} c_{n, r}^{k, m}\left(n^{\prime}, r^{\prime}\right) e\left(n^{\prime} \tau+r^{\prime} z\right)
$$

and

$$
\begin{aligned}
c_{n, r}^{k, m}\left(n^{\prime}, r^{\prime}\right)=\delta_{m} & \left(n, r, n^{\prime}, r^{\prime}\right)+(-1)^{k} \delta_{m}\left(n, r, n^{\prime},-r^{\prime}\right) \\
& +2 \pi i^{k} \operatorname{det}(2 m)^{-1 / 2}\left(D^{\prime} / D\right)^{k^{\prime} / 2} \sum_{c \geq 1}\left(H_{m, c}\left(n, r, n^{\prime}, r^{\prime}\right)\right. \\
& \left.+(-1)^{k} H_{m, c}\left(n, r, n^{\prime},-r^{\prime}\right)\right) J_{k^{\prime}}\left(\frac{2 \pi \sqrt{D D^{\prime}}}{c \operatorname{det}(2 m)}\right),
\end{aligned}
$$

where

$$
\begin{aligned}
D^{\prime} & :=\operatorname{det}\left(\begin{array}{cc}
2 n^{\prime} & r^{\prime} \\
r^{\prime} & 2 m
\end{array}\right), \\
\delta_{m}\left(n, r, n^{\prime}, r^{\prime}\right) & := \begin{cases}1 & \text { if } D=D^{\prime} \text { and } r \equiv r^{\prime} \bmod \mathbb{Z}^{g} \cdot 2 m, \\
0 & \text { otherwise },\end{cases} \\
H_{m, c}\left(n, r, n^{\prime}, r^{\prime}\right) & :=c^{-g / 2-1} \sum_{x, y} e_{c}\left((m[x]+r x+n) \bar{y}+n^{\prime} y+r^{\prime} x\right) e_{2 c}\left(r^{\prime} m^{-1} r^{t}\right) .
\end{aligned}
$$


In the last sum, $x$ and $y$ run over a complete set of representatives for $\mathbb{Z}^{(g, 1)} / c \mathbb{Z}^{(g, 1)}$ and $(\mathbb{Z} / c \mathbb{Z})^{*}$ respectively and $\bar{y}$ denotes an inverse of $y$ modulo $c$, while $e_{c}(z):=$ $e^{2 \pi i z / c}$ and $J_{v}$ denotes the Bessel function of order $v$.

Further, $\left\langle\phi, P_{n, r}^{k, m}\right\rangle=\lambda_{k, m, D} c_{\phi}(n, r)$, where $\langle\cdot, \cdot\rangle$ is the Petersson inner product on $J_{k, m, g}^{\text {cusp }}$, while $c_{\phi}(n, r)$ denotes the $(n, r)$ th Fourier coefficient of $\phi$ and

$$
\lambda_{k, m, D}:=\frac{2^{g\left(k^{\prime}-1\right)} \Gamma\left(k^{\prime}\right) \operatorname{det}(m)^{k^{\prime}-1 / 2}}{(2 \pi D)^{k^{\prime}}} .
$$

From Proposition 2.1, we conclude that the Poincaré series $P_{n, r}^{k, m}$ is nonzero if and only if its $(n, r)$ th Fourier coefficient $c_{n, r}^{k, m}$ is positive. So it is enough to prove that $c_{n, r}^{k, m}$ is nonzero.

LEMMA 2.2. The Poincaré series $P_{n, r}^{k, m}$ vanishes if $k$ is odd and $2 r \equiv 0 \bmod \mathbb{Z}^{g} \cdot 2 m$.

PROOF. Suppose that $2 r \equiv 0 \bmod \mathbb{Z}^{g} \cdot 2 m$. Then $m^{-1} r^{t} \in \mathbb{Z}^{g}$, and we may consider the group element

$$
\gamma_{m, r}:=\left(\left(\begin{array}{cc}
-1 & 0 \\
0 & -1
\end{array}\right),\left(m^{-1} r^{t}, 0\right)\right) \in \Gamma_{g}^{J}
$$

We note that

$$
\left.e(n \tau+r z)\right|_{k, m} \gamma_{m, r}=(-1)^{k} e(n \tau+r z) .
$$

It follows that $P_{n, r}^{k, m}=(-1)^{k} P_{n, r}^{k, m}$, and the proof of the lemma is complete.

We also note that the $(n, r)$ th coefficient $c(n, r)$ of a general Jacobi form of degree $g$ is zero if $k$ is odd when $2 r \equiv 0 \bmod \mathbb{Z}^{g} \cdot 2 m$. This is an easy consequence of the transformation property of Jacobi forms. See, for instance, [4] for the case where $g=1$. For the rest of this paper, we suppose that $k$ is even when $2 r \equiv 0 \bmod \mathbb{Z}^{g} \cdot 2 m$.

Now $2 m$ is a positive definite matrix with integer entries, and so $\operatorname{det}(2 m) \geq 1$, so we deduce from the Fourier expansion of $P_{n, r}^{k, m}$ that, in order to prove that $P_{n, r}^{k, m}$ is nonzero, it is enough to prove that $|S(n, r)|<(2 \pi)^{-1}$, where

$$
\begin{aligned}
S(n, r):=\operatorname{det} & (2 m)^{-1 / 2} \sum_{c \geq 1}\left(H_{m, c}(n, r, n, r)\right. \\
& \left.+(-1)^{k} H_{m, c}(n, r, n,-r)\right) J_{k^{\prime}}\left(\frac{2 \pi D}{c \operatorname{det}(2 m)}\right) .
\end{aligned}
$$

We will need the following estimates:

$$
\begin{gathered}
\left|J_{v}(x)\right| \leq \min \left\{1, \frac{1}{\Gamma(v+1)}\left(\frac{x}{2}\right)^{v}\right\} \quad \forall x>0, v \geq 2, \\
\left|H_{m, c}(n, r, n, \pm r)\right| \leq 2^{\omega(c)} c^{g / 2-1} \operatorname{gcd}(D, c),
\end{gathered}
$$

where $\omega(c)$ is the number of distinct prime divisors of $c$. See [1, 12, 13] respectively for the details. 


\section{Some simple bounds}

In this section, we will first establish the following proposition and lemma, using trivial estimates of Bessel functions.

PROPOSITION 3.1. There exists an integer $k_{0}$ such that the $(n, r)$ th Poincaré series $P_{n, r}^{k, m}$ does not vanish identically whenever $k \geq k_{0},(n, r) \in \mathbb{Z}^{+} \times \mathbb{Z}^{g}$ and e $\pi D \leq$ $k^{\prime} \operatorname{det}(2 m)$. If $k>g+3$, then one may take $k_{0}=\max \left(g+4,\left\lfloor\frac{1}{2} g\right\rfloor+69\right)$.

The Poincaré series $P_{n, r}^{k, m}$ does not vanish identically whenever $\pi D<\operatorname{det}(2 m)$ and either $k>g+3$ if $g \geq 2$ or $k>5$ if $g=1$.

LEMMA 3.2. When $g=1$, for all positive integers $n$ no greater than $(m+3) / 36$, there exists $r$ such that $r^{2}<4 m n$ and $\pi D<\operatorname{det}(2 m)$. So the condition $\pi D<\operatorname{det}(2 m)$ in the second part of Proposition 3.1 is nontrivial.

Proof. Suppose that $D<2 m / \pi<2 m / 3$. Then $2 m(2 n-1 / 3)<r^{2}<4 m n$. Note that there is an integral square in the interval $[x, y]$ when $2 \sqrt{x}+1<y-x$, so we need

$$
2\left(2 m\left(2 n-\frac{1}{3}\right)\right)^{1 / 2}+1<\frac{2}{3} m,
$$

that is,

$$
n<\frac{(2 m+3)^{2}}{144 m}=\frac{m+3}{36}+\frac{1}{16 m},
$$

which, since $n$ is an integer, is equivalent to requiring that

$$
n \leq \frac{m+3}{36} .
$$

Thus, in the case where $g=1$, the Poincaré series $P_{k, m}^{n, r}$ does not vanish identically when $k>4$ and $n \leq(m+3) / 36$.

Proof of Proposition 3.1. Let $S:=\pi D / \operatorname{det}(2 m)$. In a straightforward manner, using estimates (2.2) and (2.3), we get

$$
|S(n, r)| \leq \frac{2 S^{k^{\prime}}}{\Gamma\left(k^{\prime}+1\right)} \sum_{c \geq 1} \frac{2^{\omega(c)}}{c^{k-g-1}} .
$$

Recall Stirling's formula,

$$
n !=\sqrt{2 \pi n}(n / e)^{n} e^{\lambda_{n}},
$$

where $(12 n+1)^{-1}<\lambda_{n}<(12 n)^{-1}$ for all $n \in \mathbb{Z}^{+}$. We let $\xi:=\sum_{c \geq 1} 2^{\omega(c)} / c^{k-g-1}$. Since $\Gamma(x)$ and $(x / e)^{x}$ are increasing functions of $x$ in the intervals $\left[\frac{3}{2}, \infty\right)$ and $(1, \infty)$ respectively and $k^{\prime} \geq \frac{5}{2}$, the hypothesis of the theorem gives

$$
|S(n, r)| \leq \frac{2 \xi}{\Gamma\left(\left\lfloor k^{\prime}\right\rfloor+1\right)}\left(\frac{\left\lfloor k^{\prime}\right\rfloor+1}{e}\right)^{\left\lfloor k^{\prime}\right\rfloor+1}<\frac{2 \xi}{\sqrt{2 \pi\left(\left\lfloor k^{\prime}\right\rfloor+1\right)}} .
$$


Therefore by choosing $k$ large enough, we get the first assertion of Proposition 3.1. For the second assertion, when $k \geq g+4$, note that $\xi<1 / \pi^{2}$, which follows from the trivial estimate $2^{\omega(c)} \leq c$.

For the second part of Proposition 3.1, we refer to equation (3.1). In this case, $S<1$. We need to have $\Gamma\left(k^{\prime}+1\right)>\frac{2}{3} \pi^{2}$, since $\xi<\zeta(2)$ from the conditions on $k$. The result follows by noting that $k^{\prime} \geq 5$.

\section{Poincaré series for small weights}

When $\operatorname{Re}(s)>\frac{1}{2}\left(1-k^{\prime}\right)$, the Jacobi Poincaré series is defined using the 'Hecke trick' as in [2] by

$$
P_{n, r ; s}^{k, m}(\tau, z):=\left.\sum_{\gamma \in \Gamma_{g, \infty}^{J} \backslash \Gamma_{g}^{J}}\left(\frac{v}{|c \tau+d|^{2}}\right)^{s} e(n \tau+r z)\right|_{k, m} \gamma(\tau, z)
$$

for all $\tau=u+i v \in \mathcal{H}$, all $z \in \mathbb{C}^{g}$, and all $s \in \mathbb{C}$. If $k>\frac{1}{2} g+2$, then $P_{n, r ; 0}^{k, m} \in J_{k, m}^{\text {cusp }}$ and has the same Fourier properties as $P_{n, r}^{k, m}$. We also consider conditions on its nonvanishing in the following proposition.

PROPOSITION 4.1. There exists an integer $c(m)$ such that the Poincaré series $P_{n, r ; 0}^{k, m}$ does not vanish identically when $k \geq \max \left\{c(m), \frac{1}{2}(g+7)\right\}$ while $(n, r) \in \mathbb{Z}^{+} \times \mathbb{Z}^{g}$ and $e \pi D \leq k^{\prime} \operatorname{det}(2 m)$.

PROOF. This proposition follows from the arguments of the proof of Proposition 3.1. Here we use the following estimate for Kloosterman sums of degree $g$ (see [1, pp. 508 and 512]):

$$
H_{m, c}(n, r, n, \pm r) \leq 2^{\omega(c)} c^{-1 / 2} \operatorname{gcd}(D, c) \quad \forall c \geq C(m),
$$

where $C(m)$ is a constant. As in the proof of Proposition 3.1, put $S:=\pi D / \operatorname{det} 2 m$, and recall the definition $(2.1)$ of $S(n, r)$. Then for some positive constant $C_{1}(m)$,

$$
\begin{aligned}
|S(n, r)| \leq & \sum_{1 \leq c \leq C(m)} \frac{2^{\omega(c)+1} c^{g / 2-1} \operatorname{gcd}(D, c)}{\Gamma\left(k^{\prime}+1\right)}\left(\frac{S}{c}\right)^{k^{\prime}} \\
& +\sum_{c>C(m)} \frac{2^{\omega(c)+1} c^{-1 / 2} \operatorname{gcd}(D, c)}{\Gamma\left(k^{\prime}+1\right)}\left(\frac{S}{c}\right)^{k^{\prime}} \\
\leq & C(m)^{(g-1) / 2} \sum_{1 \leq c \leq C(m)} \frac{2^{\omega(c)+1} c^{-1 / 2} \operatorname{gcd}(D, c)}{\Gamma\left(k^{\prime}+1\right)}\left(\frac{S}{c}\right)^{k^{\prime}} \\
& +\sum_{c>C(m)} \frac{2^{\omega(c)+1} c^{-1 / 2} \operatorname{gcd}(D, c)}{\Gamma\left(k^{\prime}+1\right)}\left(\frac{S}{c}\right)^{k^{\prime}} \\
\leq & \frac{2 C_{1}(m) S^{k^{\prime}}}{\Gamma\left(k^{\prime}+1\right)} \sum_{c} \frac{2^{\omega(c)}}{c^{k^{\prime}-1 / 2}} .
\end{aligned}
$$


The condition $k>(g+7) / 2$ precisely guarantees convergence of the series above. The rest of the proof is identical to that of Proposition 3.1.

\section{Proof of Theorem 1.1}

We now come to the main result of this paper. For convenience of notation we will drop $(k, m)$ in the calculations.

Proof OF TheOREM 1.1. We use Rankin's method, as in [9]. With $S(n, r)$ as in (2.1), we need to prove that $|S(n, r)|<1 / 2 \pi$. Define

$$
\sigma:=k^{\prime-1 / 6}, \quad Q^{*}:=\frac{2 \pi D}{k^{\prime} \operatorname{det}(2 m)}, \quad M(D):=\exp \left(\frac{B_{1} \log D}{\log \log 2 D}\right),
$$

and

$$
H_{m, c}^{ \pm}(n, r, n, r):=H_{m, c}(n, r, n, r)+(-1)^{k} H_{m, c}(n, r, n,-r) .
$$

Then

$$
|S(n, r)| \leq \operatorname{det}(2 m)^{-1 / 2}\left|S_{1}(n, r)\right|+\operatorname{det}(2 m)^{-1 / 2}\left|S_{2}(n, r)\right|,
$$

where

$$
\begin{aligned}
& \left|S_{1}(n, r)\right|:=\sum_{1 \leq c \leq Q^{*}}\left|H_{m, c}^{ \pm}(n, r, n, r)\right|\left|J_{k^{\prime}}\left(\frac{k^{\prime} Q^{*}}{c}\right)\right|, \\
& \left|S_{2}(n, r)\right|:=\sum_{c>Q^{*}}\left|H_{m, c}^{ \pm}(n, r, n, r)\right|\left|J_{k^{\prime}}\left(\frac{k^{\prime} Q^{*}}{c}\right)\right| .
\end{aligned}
$$

After calculations similar to [9] (see also [7]), we get

$$
\begin{aligned}
\left|S_{1}(n, r)\right| & \leq A_{1} M(D) Q^{* g / 2-1} \sum_{d \mid D, d \leq Q^{*}} 2^{\omega(d)}\left(Q^{*} \sigma^{3}+3 d \sigma^{2}\right) \\
& \leq A_{2} M(D)^{3} \frac{Q^{* g / 2}}{k^{\prime 1 / 2}}+A_{3} M(D)^{3} \frac{Q^{* g / 2}}{k^{\prime / 3}} \\
& \leq A_{4} M(D)^{3} \frac{(\pi D)^{g / 2}}{\operatorname{det}(2 m)^{g / 2} k^{\prime g / 2+1 / 2}}+A_{5} M(D)^{3} \frac{(\pi D)^{g / 2}}{\operatorname{det}(2 m)^{g / 2} k^{\prime g / 2+1 / 3}}
\end{aligned}
$$

However, the sum $S_{2}(n, r)$ needs to be handled differently. We have

$$
\begin{aligned}
\left|S_{2}(n, r)\right| \leq & \sum_{Q^{*}<c \leq k^{\prime} Q^{*}} 2^{\omega(c)+1} c^{g / 2-1} \operatorname{gcd}(D, c)\left|J_{k^{\prime}}\left(\frac{2 \pi D}{c \operatorname{det}(2 m)}\right)\right| \\
& +\sum_{c>k^{\prime} Q^{*}} 2^{\omega(c)+1} c^{g / 2-1} \operatorname{gcd}(D, c)\left|J_{k^{\prime}}\left(\frac{2 \pi D}{c \operatorname{det}(2 m)}\right)\right|
\end{aligned}
$$




$$
\begin{aligned}
& \leq 2 M(D) \frac{(\pi D)^{k^{\prime}}}{\operatorname{det}(2 m)^{k^{\prime}} \Gamma\left(k^{\prime}+1\right)} \sum_{Q^{*}<c \leq k^{\prime} Q^{*}} \frac{1}{c^{k^{\prime}-g / 2}} \\
& \quad+2 \sum_{c>k^{\prime} Q^{*}} c^{g / 2}\left|J_{k^{\prime}}\left(\frac{2 \pi D}{c \operatorname{det}(2 m)}\right)\right| \\
& \leq \frac{2 M(D)}{Q^{* k^{\prime}-g / 2-1-\epsilon}} \frac{(\pi D)^{k^{\prime}}}{\operatorname{det}(2 m)^{k^{\prime}} \Gamma\left(k^{\prime}+1\right)} \sum_{Q^{*}<c \leq k^{\prime} Q^{*}} \frac{1}{c^{1+\epsilon}} \\
& \quad+\frac{2(\pi D)^{g / 2+1+\delta}}{\operatorname{det}(2 m)^{k^{\prime}} \Gamma\left(k^{\prime}+1\right)} \sum_{c>k^{\prime} Q^{*}} \frac{1}{c^{1+\delta}} \\
& \leq a_{0} \frac{M(D)}{k^{\prime g / 2+3 / 2+\epsilon}}\left(\frac{\pi D}{\operatorname{det}(2 m)}\right)^{g / 2+1+\epsilon}+a_{1} \frac{(\pi D / \operatorname{det}(2 m))^{g / 2+1+\delta}}{k^{\prime k^{\prime}+1 / 2}},
\end{aligned}
$$

where the $a_{i}$ and $A_{j}$ are constants, and $0<\epsilon, \delta<1$. Now let $\alpha(g)=2(3 g+2)^{-1}$. For all $g \geq 1$, choose $0<\epsilon, \delta<1 / 2$. We find that $S_{1}(n, r)$ and $S_{2}(n, r)$ are small if $k$ is chosen large enough. If $g \geq 5$, then we find that a better bound, $\alpha(g)=2(3 g)^{-1}$, also works. This completes the proof.

\section{An explicit basis for $J_{k, m}^{\text {cusp }}$ and the proof of Theorem 1.2}

It is well known that the space of cusp forms $S_{k}$ for $\operatorname{SL}(2, \mathbb{Z})$ has an explicit basis of the form $\left\{\Delta E_{4}^{a} E_{6}^{b} \mid 4 a+6 b=k-12\right\}$. Here $\Delta$ is the discriminant cusp form of weight 12 , while $E_{4}$ and $E_{6}$ are the Eisenstein series of weight 4 and 6 . Such an explicit result is not available for $J_{k, m}^{\text {cusp }}$. Petersson proved that the set of Poincaré series $\left\{P_{1}^{k}, \ldots, P_{d_{k}}^{k}\right\}$ is a basis for $S_{k}$, where $d_{k}=\operatorname{dim} S_{k}$. We prove the corresponding result for Jacobi forms. The proof is based on the dimension formula in [4, p. 121].

THEOREM 6.1. Let $k \geq m+12$, and define

$$
D_{\mu}:=4 m\left(\left\lfloor\frac{\mu^{2}}{4 m}\right\rfloor+1\right)-\mu^{2} .
$$

Then $\left\{P_{D_{\mu}+4 m \lambda_{\mu}, \mu}^{k, m}\right\}$ is the classical basis for $J_{k, m, 1}^{\text {cusp }}$, where the range of the indices is given as follows:

(1) if $k$ is even, $\mu=0,1, \ldots, m ; \lambda_{\mu}=0,1, \ldots, \operatorname{dim} S_{k+2 \mu}-\left\lfloor\mu^{2}(4 m)^{-1}\right\rfloor-1$;

(2) if $k$ is odd, $\mu=1, \ldots, m-1 ; \lambda_{\mu}=0,1, \ldots, \operatorname{dim} S_{k+2 \mu-1}-\left\lfloor\mu^{2}(4 m)^{-1}\right\rfloor$ ' -1 .

PROOF. We prove the theorem for even $k$, the odd case being analogous. The condition that $k \geq m+12$ ensures that $\operatorname{dim} S_{k+2 \mu} \geq\left\lfloor\mu^{2}(4 m)^{-1}\right\rfloor+1$ (see [4, p. 103]). The proof follows Petersson's argument in the elliptic case (see [8, 10]). Let $d_{k, m}=$ 
$\operatorname{dim} J_{k, m}^{\text {cusp }}$ and $\left\{\phi_{1}, \ldots, \phi_{d_{k, m}}\right\}$ be an orthonormal basis. We write

$$
\phi_{j}(\tau, z)=\sum_{\substack{D^{\prime}>0, r \in \mathbb{Z} \\ D^{\prime} \equiv-r^{2} \bmod 4 m}} c_{j}\left(D^{\prime}, r\right) e\left(\frac{D^{\prime}+r^{2}}{4 m} \tau+r z\right)
$$

and

$$
P_{D_{\mu}+4 m \lambda_{\mu}, \mu}^{k, m}=\lambda_{k, m, D_{\mu}+4 m \lambda_{\mu}}^{-1} \sum_{j=1}^{d_{k, m}} c_{j}\left(D_{\mu}+4 m \lambda_{\mu}, \mu\right) \phi_{j},
$$

where $\mu$ and $\lambda_{\mu}$ vary as in the statement of the theorem. We get a $d_{k, m} \times d_{k, m}$ matrix indexed by pairs $\left(D_{\mu}+4 m \lambda_{\mu}, \lambda_{\mu}\right)$ and $j$. It suffices to prove that the matrix is invertible. If not, then there would be a linear relation

$$
\sum_{j=1}^{d_{k, m}} \xi_{j} c_{j}\left(D_{\mu}+4 m \lambda_{\mu}, \mu\right)=0
$$

for all $\left(D_{\mu}+4 m \lambda_{\mu}, \mu\right)$, where $\left(\xi_{1}, \ldots, \xi_{d_{k, m}}\right) \neq(0, \ldots, 0)$.

Considering the nonzero Jacobi cusp form $\Phi:=\sum_{j=1}^{d_{k, m}} \xi_{j} \phi_{j}$, we see that the Fourier coefficients $c_{\Phi}\left(D_{\mu}+4 m \lambda_{\mu}, \mu\right)$ of $\Phi$ are zero, where $\mu$ and $\lambda_{\mu}$ vary as in the theorem. This implies that $D_{2 \mu} \Phi=0$ when $\mu=0, \ldots, m$ (see [4, p. 32] for the definition of the operators $D_{2 \mu}$ ), which shows that $\Phi=0$, a contradiction.

To see that $D_{2 \mu} \Phi=0$ when $\mu=0, \ldots, m$, we recall the following Fourier expansion of the modular form $D_{2 v} \Phi$ of weight $k+2 v$, where $k$ is even and $v=$ $0, \ldots, m$ (see $[4$, p. 32]):

$$
D_{2 v} \Phi=A_{k, v} \sum_{n \geq 0} \sum_{\substack{r: r^{2}<4 m n \\ \mu: 0 \leq \mu \leq v}} \frac{(k+2 v-\mu-2) !(-m n)^{\mu} r^{2 v-2 \mu}}{(k+2 v-2) ! \mu !(2 v-2 \mu) !} c_{\Phi}\left(n_{v}, r_{v}\right) q^{n}
$$

where $q:=e(\tau)$ and

$$
A_{k, v}:=(2 \pi i)^{-v} \frac{(k+2 v-2) !(2 v) !}{(k+v-2) !} .
$$

Let $\ell$ be an even positive integer, and put $d_{\ell}:=\operatorname{dim} S_{\ell}$. It is well known that an elliptic cusp form $\sum_{n=1}^{\infty} a(n, f) q^{n}$ of weight $\ell$ is determined by the first $d_{\ell}$ of its Fourier coefficients $a(1, f), \ldots, a\left(d_{\ell}, f\right)$. Therefore, looking at equation (6.1), we need to prove that

$$
c_{\Phi}\left(n_{v}, r_{v}\right)=0
$$

for all $v=0, \ldots, m$, all $r_{v}$ such that $r_{v}^{2}<4 m n_{v}$ and $0 \leq r_{v} \leq m$, and all $n_{v}$ such that $\left\lfloor r_{v}^{2}(4 m)^{-1}\right\rfloor+1 \leq n_{v} \leq d_{k+2 v}$. Let $\ell$ denote $k+2 v$, where $v=0, \ldots, m$, and for convenience, we drop the suffix $v$ in the terms $n_{v}$ and $r_{v}$. To see (6.2), if $|r|>m$ in 
equation (6.1), we can find suitable integers $x$ and $n^{\prime} \geq 1$ such that

$$
-m \leq r^{\prime}=r-2 m x \leq m \text { and } 4 m n^{\prime}-r^{\prime 2}=4 m n-r^{2} .
$$

We then use the facts that $c_{\Phi}\left(n^{\prime}, r^{\prime}\right)=c_{\Phi}(n, r)$ and $n \geq n^{\prime} \geq 1$, implying that $n^{\prime}$ satisfies the same upper bound as $n$, namely, $\left\lfloor r^{\prime 2}(4 m)^{-1}\right\rfloor+1 \leq n^{\prime} \leq d_{\ell}$. We may finally reduce to the case where $0 \leq r \leq m$ since $c_{\Phi}(n, r)=c_{\Phi}(n,-r)$ as $\ell$ is even.

But any such $n_{v}$ may be written

$$
n_{v}=\left\lfloor v^{2}(4 m)^{-1}\right\rfloor+1+\lambda_{v}=\frac{D_{v}+v^{2}+4 m \lambda_{v}}{4 m},
$$

where $0 \leq v \leq m$ and $D_{v}$ and $\lambda_{v}$ are as in the statement of the theorem. This completes the proof.

The Eichler-Zagier map $Z_{1}: J_{k, 1} \rightarrow M_{k-1 / 2}^{+}$for Jacobi forms of integral weight and index $1\left(M_{k-1 / 2}^{+}\right.$denotes the Kohnen + space for $\Gamma_{0}(4)$, as in [6] $)$ is defined by

$$
Z_{1}: \sum_{\substack{D \in \mathbb{Z}^{+}, r \in \mathbb{Z} \\ D \equiv-r^{2} \bmod 4}} c(D) e\left(\frac{D+r^{2}}{4} \tau+r z\right) \mapsto \sum_{D \in \mathbb{Z}^{+}} c(D) e(D \tau),
$$

where the Fourier coefficient $c(D)$ does not depend on $r$.

Suppose that $k$ is even and $(-1)^{k-1} D \equiv 0,1 \bmod 4$. Following the notation in [6], let $P_{k-1,4, D}$ be the $D$ th Poincaré series in $M_{k-1 / 2}^{+}$. By comparing the Fourier developments of $P_{D, r}$ from [1] and of $P_{k-1,4, D}$ from [6], we get the following result.

Proposition 6.2. The Eichler-Zagier map $Z_{1}$ takes $P_{D, r} \in J_{k, 1}^{\text {cusp }}$ to $3 P_{k-1,4, D} \in$ $M_{k-1 / 2}^{+}$.

PROOF. The proof involves an easy calculation of Gauss sums, which may be found in [3].

PROpOsition 6.3. There exist positive constants $k_{0}$ and $B$, where $B>4 \log 2$, such that, for all even $k \geq k_{0}$ and all positive integers $D \leq k^{2} \exp (-B \log k / \log \log k)$, the Poincaré series $P_{k-1,4, D}$ and hence also the Poincaré series $P_{D, r}^{k, 1}$ do not vanish identically.

PROOF. From the Fourier expansion of $P_{k-1,4, D}$ given in [6], we see that the proof is the same as in the case of integral weight Poincaré series for congruence subgroups of $\operatorname{SL}(2, \mathbb{Z})$ given in [7]; we omit it. 
Proof OF THEOREM 1.2. We write $S(n, r)=S_{1}(n, r)+S_{2}(n, r)$, where

$$
\begin{aligned}
& S_{1}(n, r):=i^{k} \pi \sqrt{2} m^{-1 / 2} \sum_{1 \leq c \leq \pi D / m} H_{m, c}^{ \pm}(n, r, n, r) J_{k^{\prime}}\left(\frac{\pi D}{m c}\right), \\
& S_{2}(n, r):=i^{k} \pi \sqrt{2} m^{-1 / 2} \sum_{c>\pi D / m} H_{m, c}^{ \pm}(n, r, n, r) J_{k^{\prime}}\left(\frac{\pi D}{m c}\right) .
\end{aligned}
$$

To estimate $S_{1}(n, r)$, we use the following estimate of Bessel functions:

$$
\left|J_{v}(r)\right| \leq C r^{-1 / 3},
$$

when $v \geq 0$ and $r \geq 1$; see [5, Lemma 3.4]. The constant $C$ in this lemma may be computed to be the constant $A$ in Theorem 1.2 of this paper using [11, p. 333]. Then

$$
\begin{aligned}
\left|S_{1}(n, r)\right| & \leq \frac{2^{3 / 2} \pi}{m^{1 / 2}} \sum_{1 \leq c \leq \pi D / m} \frac{2^{\omega(c)} \operatorname{gcd}(D, c)}{c^{1 / 2}}\left|J_{k^{\prime}}\left(\frac{\pi D}{m c}\right)\right| \\
& \leq \frac{2^{3 / 2} m^{1 / 3} \pi^{2 / 3}}{D^{1 / 3} m^{1 / 2}} M(\pi D / m) \sum_{1 \leq c \leq \pi D / m} \frac{\operatorname{gcd}(D, c)}{c^{1 / 6}} \\
& \leq \frac{2^{3 / 2} \pi^{2 / 3}}{D^{1 / 3} m^{1 / 6}} M(\pi D / m) \sum_{d \mid D, d<\pi D / m} d \\
& \leq \frac{2^{3 / 2} D^{2 / 3} \pi^{5 / 3}}{m^{7 / 6}} M(\pi D / m) \sigma_{0}(D),
\end{aligned}
$$

and

$$
\begin{aligned}
\left|S_{2}(n, r)\right| & \leq \frac{2^{3 / 2} \pi}{m^{1 / 2}} \sum_{c>\pi D / m} c^{3 / 2}\left|J_{k^{\prime}}\left(\frac{\pi D}{m c}\right)\right| \\
& \leq \frac{2^{3 / 2} \pi}{\Gamma\left(k^{\prime}+1\right) m^{1 / 2}} \sum_{c>\pi D / m} c^{3 / 2}\left(\frac{\pi D}{m c}\right)^{3 / 2+2} \\
& \leq \frac{2^{3 / 2} \pi^{9 / 2} D^{7 / 2}}{\Gamma\left(k^{\prime}+1\right) m^{4}} \sum_{c>\pi D / m} \frac{1}{c^{2}} \\
& \leq \frac{2^{3 / 2} \pi^{13 / 2} D^{7 / 2}}{6 \Gamma\left(k^{\prime}+1\right) m^{4}} .
\end{aligned}
$$

From the bound given in Theorem 1.2, it follows from estimates (6.4) and (6.5) that $S_{1}$ and $S_{2}$ are both less than $\frac{1}{2}$ in absolute value. Finally, from the expression for the $(n, r)$ th Fourier coefficient of $P_{n, r}^{k, m}$ given in Proposition 2.1, we get the theorem. 


\section{Further results}

Recall the one-dimensional Kloosterman sum for a positive integer $c$,

$$
S(r, m ; c)=\sum_{\substack{1 \leq h \leq c \\(h, c)=1}} e_{c}\left(r h+m h^{\prime}\right),
$$

where $h h^{\prime} \equiv 1 \bmod c$. It is well known (see, for example, [9, Section 3]) that the following relation holds for a prime $p$ :

$$
S\left(r p^{\rho}, m p^{\mu} ; c p\right)=S\left(r, m p^{\rho+\mu} ; c p\right)+p S\left(r p^{\rho-1}, m p^{\mu-1} ; c\right),
$$

where $p \nmid r m$ and $\rho, \mu \geq 1$.

Definition 7.1. Suppose that $n, n^{\prime} \in \mathbb{Z}^{+}$and $r, r^{\prime} \in \mathbb{Z}^{g}$. We let

$$
\begin{aligned}
K_{m, c}\left(n, r, n^{\prime}, r^{\prime}\right) & =\sum_{x, y} e_{c}\left((m[x]+r x+n) \bar{y}+n^{\prime} y+r^{\prime} x\right) \\
& =c^{g / 2+1} e_{2 c}\left(-r^{\prime} m^{-1} r^{t}\right) H_{m, c}\left(n, r, n^{\prime}, r^{\prime}\right),
\end{aligned}
$$

where in the sum, $x$ and $y$ run over a complete set of representatives for $\mathbb{Z}^{(g, 1)} / c \mathbb{Z}^{(g, 1)}$ and $(\mathbb{Z} / c \mathbb{Z})^{*}$ respectively and $\bar{y}$ denotes an inverse of $y$ modulo $c$.

Lemma 7.2. Let $p$ be a odd prime such that $p \mid(m, r)$ and $p \nmid n n^{\prime}$, and let $\mu^{\prime}=$ $\mu-1$ and $\rho^{\prime}=\rho-1$. Then the following identity holds:

$$
\begin{aligned}
K_{m p^{\mu}, c p}\left(p^{\mu} n, p^{\mu} r, p^{\rho} n^{\prime}, r^{\prime} p\right)= & K_{m p^{\rho+\mu}, c p}\left(p^{\rho+\mu} n, p^{\rho+\mu} r, n^{\prime}, r^{\prime} p\right) \\
& +p^{2} K_{m p^{\mu^{\prime}, c}}\left(p^{\left.\mu^{\prime} n, p^{\mu^{\prime}} r, p^{\rho^{\prime}} n^{\prime}, r^{\prime}\right) .} .\right.
\end{aligned}
$$

Proof. The proof follows by noting that

$$
K_{m, c p}\left(n, r, n^{\prime}, r^{\prime} p\right)=\sum_{x \bmod c p} e_{c}\left(r^{\prime} x\right) S\left(n^{\prime}, m[x]+r x+n ; c p\right),
$$

from which the left-hand side and the first term on the right-hand side in (7.3) are taken care of by summing both sides of equation (7.2) with appropriate arguments over $x$ modulo $c p$. For the last term, we split the summation in equation (7.4) as $x=c x_{1}+x_{2}$, where $x_{1}$ and $x_{2}$ range over $\mathbb{Z}^{g} / p \mathbb{Z}^{g}$ and $\mathbb{Z}^{g} / c \mathbb{Z}^{g}$, respectively. After replacing $\left(m, n, r, n^{\prime} ; c p\right)$ by $\left(p^{\mu^{\prime}} m, p^{\mu^{\prime}} n, p^{\mu^{\prime}} r, p^{\rho^{\prime}} n^{\prime} ; c\right)$ and summing, we have

$$
\begin{aligned}
& \sum_{x \bmod c} e_{c p}\left(r^{\prime} x\right) S\left(p^{\rho^{\prime}} n^{\prime}, p^{\mu^{\prime}}(m[x]+r x+n) ; c\right) \\
& \quad=\sum_{x_{1}, x_{2}} e_{c}\left(r^{\prime}\left(c x_{1}+x_{2}\right)\right) S\left(p^{\rho^{\prime}} n^{\prime}, p^{\mu^{\prime}}\left(m\left[c x_{1}+x_{2}\right]+p^{\mu^{\prime}}\left(r\left(c x_{1}+x_{2}\right)+n\right)\right) ; c\right) \\
& \quad=\sum_{x_{1}} e\left(r^{\prime} c x_{1}\right) \sum_{x_{2}} e_{c}\left(r^{\prime}, x_{2}\right) S\left(p^{\rho^{\prime}} n^{\prime}, p^{\mu^{\prime}}\left(m\left[x_{2}\right]+r x_{2}+n\right) ; c\right) \\
& =p K_{m p^{\mu^{\prime}, c}}\left(p^{\mu^{\prime}} n, p^{\mu^{\prime}} r, p^{\rho^{\prime}} n^{\prime}, r^{\prime}\right) .
\end{aligned}
$$

Therefore the lemma follows from (7.2). 
Proof of Theorem 1.3. From Lemma 7.2, we easily deduce that under the conditions of the lemma,

$$
\begin{aligned}
& H_{m p^{\mu}, c p}\left(p^{\mu} n, p^{\mu} r, p^{\rho} n^{\prime}, r^{\prime} p\right) \\
& =H_{m p^{\rho+\mu}, c p}\left(p^{\rho+\mu_{n}}, p^{\rho+\mu} r, n^{\prime}, r^{\prime} p\right) \\
& \quad+p^{-g / 2+1} H_{m p^{\mu-1}, c}\left(p^{\mu-1} n, p^{\mu-1} r, p^{\rho-1} n^{\prime}, r^{\prime}\right) .
\end{aligned}
$$

The following equality also follows from the definition, when $p \nmid c$ :

$$
H_{m p^{\mu}, c}\left(p^{\mu} n, p^{\mu} r, p^{\rho} n^{\prime}, r^{\prime} p\right)=H_{m p^{\rho+\mu}, c}\left(p^{\rho+\mu} n, p^{\rho+\mu} r, n^{\prime}, r^{\prime} p\right) .
$$

We sum equation (7.5) over all $c \geq 1$, and equation (7.6) over all $c \geq 1$ coprime to $p$, after multiplying them by the appropriate Bessel functions, and add them; see Proposition 2.1. Gathering all of the terms, putting $\rho=\mu$ and $n^{\prime}=n$ and $r^{\prime} p=p^{\mu} r$, in all three sums, we find positive constants $\alpha_{1}$ and $\alpha_{2}$ such that

$$
c^{k, p^{\mu} m}\left(p^{\mu} n, p^{\mu} r\right)=\alpha_{1} c^{k, p^{2 \mu} m}\left(p^{2 \mu} n, p^{2 \mu} r ; n, p^{\mu} r\right)+\alpha_{2} c^{k, p^{\mu-1} m}\left(p^{\mu-1} n, p^{\mu-1} r\right)
$$

(we have used the notation $\left.c_{P_{n, r}^{k, m}}(n, r):=c^{k, m}(n, r ; n, r)=c^{k, m}(n, r)\right)$. This immediately implies the conclusion of Theorem 1.3.

\section{Acknowledgements}

The author would like to thank the referee for his comments on the paper. The author also wishes to thank Professor B. Ramakrishnan for going through the manuscript and for his support and encouragement. This paper is a part of the author's $\mathrm{PhD}$ thesis at the Harish Chandra Research Institute, Allahabad, India.

\section{References}

[1] S. Böcherer and W. Kohnen, 'Estimates for Fourier coefficients of Siegel cusp forms', Math. Ann. 297 (1993), 499-517.

[2] K. Bringmann and T. Yang, 'On Jacobi Poincaré series of small weight', Int. Math. Res. Not. (2007), 21.

[3] S. Das, 'Nonvanishing of Jacobi Poincaré series', arXiv, http://arxiv.org/abs/0910.4303v2.

[4] M. Eichler and D. Zagier, The Theory of Jacobi Forms, Progress in Mathematics, 55 (Birkhäuser, Boston, 1985).

[5] K. Guo, 'A uniform $L^{p}$ estimate of Bessel functions and distributions supported on $S^{n-1}$, Proc. Amer. Math. Soc. 125(5) (1997), 1329-1340.

[6] W. Kohnen, 'Fourier coefficients of modular forms of half integral weight', Math. Ann. 271 (1985), 237-268.

[7] C. J. Mozzochi, 'On the nonvanishing of Poincaré series', Proc. Edinb. Math. Soc. 32 (1989), 133-137.

[8] H. Petersson, 'Über eine Metrisierung der automorphen Formen und die Theorie der Poincaréschen Reihen', Math. Ann. 117 (1940/41), 453-529.

[9] R. A. Rankin, 'The vanishing of Poincaré series', Proc. Edinb. Math. Soc. 23 (1980), 151-161.

[10] J. R. Smart, 'A basis theorem for cusp forms on groups of genus zero', Michigan Math. J. 10 (1963), 375-380. 
[11] E. M. Stein, Harmonic Analysis: Real-Variable Methods, Orthogonality, and Oscillatory Integrals (Princeton University Press, Princeton, NJ, 1993).

[12] K. Stempak, 'A weighted uniform $L^{p}$ estimate of Bessel functions: a note on a paper of Guo', Proc. Amer. Math. Soc. 128(10) (2000), 2943-2945.

[13] G. N. Watson, A Treatise on the Theory of Bessel Functions (Cambridge University Press, Cambridge, 1966).

SOUMYA DAS, School of Mathematics,

Tata Institute of Fundamental Research, Homi Bhabha Road, Mumbai-400005, India e-mail: somu@math.tifr.res.in 